\title{
Survival outcomes after adjuvant radiotherapy for aggressive fibromatosis depend on time frame and nuclear $\beta$-catenin
}

\author{
Jae Sik Kim, MD ${ }^{1}$, Hak Jae Kim, MD, PhD ${ }^{1,2}$, Me-Yeon Lee, MD, PhD ${ }^{3}$, \\ Kyung Chul Moon, MD, PhD ${ }^{4}$, Seung Geun Song, MD ${ }^{4}$, Han-Soo Kim, MD, PhD ${ }^{5}$, \\ Ilkyu Han, MD, PhD ${ }^{5}$, II Han Kim, MD, PhD ${ }^{1,2}$ \\ 'Department of Radiation Oncology, Seoul National University College of Medicine, Seoul; \\ ${ }^{2}$ Cancer Research Institute, Seoul National University, Seoul; \\ ${ }^{3}$ Department of Radiation Oncology, Hallym University College of Medicine, Seoul; \\ Departments of ${ }^{4}$ Pathology and ${ }^{5}$ Orthopaedic Surgery, Seoul National University College of Medicine, Seoul, Korea
}

\begin{abstract}
Purpose: To identify prognostic factors influencing progression-free survival (PFS) of aggressive fibromatosis (AF) after postoperative radiotherapy (PORT) and assess correlations between immunohistochemistry (IHC) features of $\beta$-catenin/smooth muscle actin (SMA) and PFS.

Materials and Methods: Records of 37 patients with AF treated by PORT from 1984 to 2015 were retrospectively reviewed. Fifteen patients underwent wide excision for AF and 22 patients received debulking operation. The median total dose of PORT was 59.4 Gy. IHC staining results of $\beta$-catenin and SMA were available for 11 and 12 patients, respectively.

Results: The median follow-up duration was 105.9 months. Five-year PFS rate was 70.9\%. Tumor size or margin status was not related to PFS in univariate analysis ( $p=0.197$ and $p=0.716$, respectively). Multivariate analysis showed that increased interval from surgery to PORT (>5.7 weeks) was a marginal risk factor for PFS ( $p=0.054)$. Administration of PORT at the initial diagnosis resulted in significantly improved PFS compared to deferring PORT after recurrence $(p=0.045)$. Patient with both risk factors of deferring PORT after recurrence and interval from surgery to PORT $>5.7$ weeks had significantly lower 5 -year PFS than patients without risk factor ( $34.1 \%$ vs. $100.0 \% ; p=0.012)$. Nuclear $\beta$-catenin intensity tended to inversely correlate with 5 -year PFS, although it did not reach statistical significance ( $62.5 \%$ at low vs. $100.0 \%$ at high; $p=0.260$ ). SMA intensity was not related to PFS $(p=0.700)$.

Conclusion: PORT should be performed immediately after surgery irrespective of margin status or tumor size especially in recurrent case. Nuclear $\beta$-catenin staining intensity of $\mathrm{IHC}$ might correlate with local recurrence.
\end{abstract}

Keywords: Aggressive fibromatosis, Adjuvant radiotherapy, Immunohistochemistry, Beta catenin, Progression-free survival

\section{Introduction}

Aggressive fibromatosis (AF) or desmoid tumor is a rare benign monoclonal myofibroblastic neoplasm [1-3]. However, it is markedly locally infiltrative with high local recurrence rate [1]. Recently updated recommendations of the European

Received 02 December 2018, Revised 02 January 2019, Accepted 18 January 2019.

Correspondence: II Han Kim, MD, PhD, Department of Radiation Oncology, Seoul National University Hospital, 101 Daehakro, Jongno-gu, Seoul 03080, Korea. Tel: +82-2-2072-2528, Fax: +82-2-765-3317, E-mail: ihkim@snu.ac.kr (http://orcid. org/0000-0002-4755-5201)

(c) This is an Open Access article distributed under the terms of the Creative Commons Attribution Non-Commercial License (http://creativecommons.org/ licenses/by-nc/4.0/) which permits unrestricted non-commercial use, distribution, and reproduction in any medium, provided the original work is properly cited.

www.e-roj.org 
Desmoid Working Group suggest a watch and wait strategy as front-line approach until as long as 2 years after diagnosis. Surgery is considered if the tumor progresses according to its anatomical location [2]. Postoperative radiotherapy (PORT) is not strongly recommended unless surgery is performed for AF in head, neck, and intrathoracic site [2]. PORT has been suggested when there are high risk features of local recurrence such as recurrent disease and positive resection margin $[4,5]$. However, treatments were not uniform among patients in previous studies. In addition, prognostic factors varied from study to study.

$\beta$-catenin is a transcriptional activator [6]. S45F mutation of $\beta$-catenin is significantly associated with an increased risk of recurrence $[3,7]$. Lazar et al. [3] have reported that immunohistochemistry (IHC) staining intensity of nuclear $\beta$-catenin is inversely correlated with recurrence. In their study, IHC staining was evaluated only in primary AF patients for whom whether PORT was performed was unknown.

Activated myofibroblasts with smooth muscle actin (SMA) are main cellular components in the tumor stroma [8]. It has been proposed that these activated myofibroblasts are associated with tumor progression [9]. Gebert et al. [10] have revealed weak positive IHC staining for SMA in less than 10\% of AF cells. However, they did not analyze the relationship between SMA and survival of patients with AF.

The objective of this study was to evaluate progression-free survival (PFS) and prognostic factors of patients with primary or recurrent AF treated with PORT. In addition, correlations of IHC markers and PFS were determined.

\section{Materials and Methods}

\section{Patients}

We retrospectively recruited 41 patients with pathologicallyproven AF who underwent PORT from 1984 to 2015 at our institution. We excluded 4 patients who received radical radiotherapy (RT). Finally, a total of 37 patients were analyzed in this study. The Institutional Review Board of Seoul National University Hospital approved this study protocol (No. H-1803036-926). Medical records of patients were retrieved from the hospital database. The informed consent was waived because of the retrospective design.

\section{Immunohistochemistry}

We reviewed all available IHC slides for $\beta$-catenin $(n=5)$ and SMA $(n=5)$. Additional IHC staining was performed if surgical specimens were stored ( $\beta$-catenin, $n=6$; SMA, $n=7)$.
Four- $\mu$ m-thick sections were stained using a BenchMark autostainer (Ventana, Tucson, AZ, USA) according to the manufacturer's instructions. Mouse monoclonal antibodies against $\beta$-catenin (CAT-5H10, dilution 1:800; Invitrogen, Carlsbad, CA, USA) and SMA (1A4, dilution 1:500; Dako, Glostrup, Denmark) were used. Intensity of IHC was graded by two independent researchers. The grading system used in the study of Lazar et al. [3] was modified to be applied for nuclear $\beta$-catenin staining. If nucleus accumulation was not observed or if it only could be confirmed by viewing at $\times 400$ low intensity was considered. Otherwise, high intensity was considered. For SMA grading, we used the method adapted by Parikh et al. [11]. If staining intensity of tumor cell was equal to or higher than that of vessels, it was defined as high intensity. Otherwise, it was defined as low intensity.

\section{Statistical analysis}

PFS was defined from the date of RT completion to the date of disease progression or recurrence. We defined RT timing as the interval from surgery to beginning of RT. RT duration was defined as the period between beginning and end of RT. PFS rates were estimated using Kaplan-Meier method and compared using log-rank test. To identify prognostic factors of PFS, univariate and multivariate analyses were conducted using Cox proportional hazard model. We conducted multivariate analysis using variables with $p<0.100$ in univariate analysis. $A$ p-value less than 0.05 was considered statistically significant. All statistical analyses were conducted using $R$ project version 3.4.2 (https://www.r-project.org/).

\section{Results}

\section{Patient characteristics}

Baseline characteristics of patients and treatment are shown in Table 1 . The median age of patients was 29 years (range, 3 to 80 years). Lower extremity was the most common site of involvement ( $n=14,37.8 \%$ ), followed by the abdomen ( $n=8,21.6 \%$ ). Fifteen patients received PORT at the time of initial diagnosis and 22 patients were treated with PORT after recurrence. Among the recurred 22 patients, the median number of recurrences before the first PORT was 2 (range, 1 to 8). Fifteen patients underwent wide excision and 29 patients had positive resection margin. PORT was delivered with twodimensional RT ( $n=19$ ), three-dimensional conformal RT ( $n=$ 16), or intensity-modulated RT technique $(n=2)$.

We applied the general RT principle to the sarcoma and included the site of the primary tumor and a range of 
Table 1. Patients' characteristics $(n=37)$

\begin{tabular}{|c|c|}
\hline Characteristic & No. (\%) \\
\hline Age (yr), median (range) & $29(3-80)$ \\
\hline \multicolumn{2}{|l|}{ Sex } \\
\hline Male & $16(43.2)$ \\
\hline Female & $21(56.8)$ \\
\hline \multicolumn{2}{|l|}{ Location } \\
\hline Neck & $5(13.5)$ \\
\hline Thorax & $3(8.1)$ \\
\hline Abdomen & $8(21.6)$ \\
\hline Back & $2(5.4)$ \\
\hline Upper extremity & $5(13.5)$ \\
\hline Lower extremity & $14(37.8)$ \\
\hline \multicolumn{2}{|l|}{ PORT at } \\
\hline Initial & $15(40.5)$ \\
\hline Recurrence & $22(59.5)$ \\
\hline \multicolumn{2}{|l|}{ Tumor size (cm) } \\
\hline$<10$ & $23(62.2)$ \\
\hline$\geq 10$ & 14 (37.8) \\
\hline \multicolumn{2}{|l|}{ Surgery } \\
\hline Wide excision & $15(40.5)$ \\
\hline Debulking & $22(59.5)$ \\
\hline \multicolumn{2}{|l|}{ Resection margin } \\
\hline egative & $8(21.6)$ \\
\hline Positive & $29(78.4)$ \\
\hline \multicolumn{2}{|l|}{ PORT modality } \\
\hline 2D-RT & $19(51.4)$ \\
\hline 3D-CRT & $16(43.2)$ \\
\hline IMRT & $2(5.4)$ \\
\hline
\end{tabular}

PORT, postoperative radiotherapy; 2D-RT, two-dimensional radiotherapy; 3D-CRT, three-dimensional conformal radiotherapy; IMRT, intensity-modulated radiotherapy.

surrounding normal tissues and treated appropriately considering the primary site of the tumor. In particular, in patients treated with three-dimensional conformal and intensity-modulated RT, clinical target volume was defined by three-dimensional auto-expansion of $2-5 \mathrm{~cm}$ in the superiorinferior direction and $1-3 \mathrm{~cm}$ in the radial direction to the tumor bed. Planning target volume was defined by threedimensional auto-expansion of $0-0.5 \mathrm{~cm}$ to the clinical target volume. The median total dose was $59.4 \mathrm{~Gy}$ (range, 45 to 66 Gy in 1.7 to $2.15 \mathrm{~Gy}$ ). The median of RT timing was 5.7 weeks (range, 3.0 to 10.9 weeks). Three patients were treated with systemic therapies: one patient received tamoxifen followed by imatinib, one received imatinib following methotrexate and vinblastine, and the other received vincristine and actinomycin-D. Two patients (5.4\%) were associated with familial adenomatous polyposis.

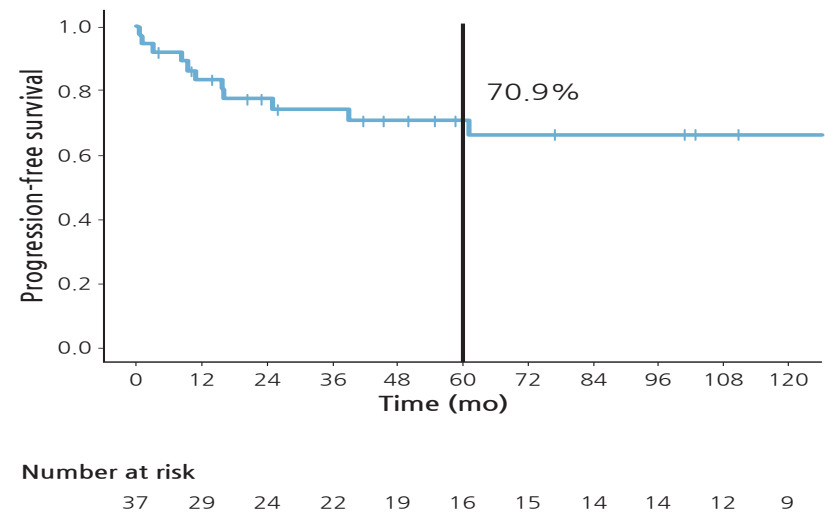

Fig. 1. Kaplan-Meier survival curve of progression-free survival.

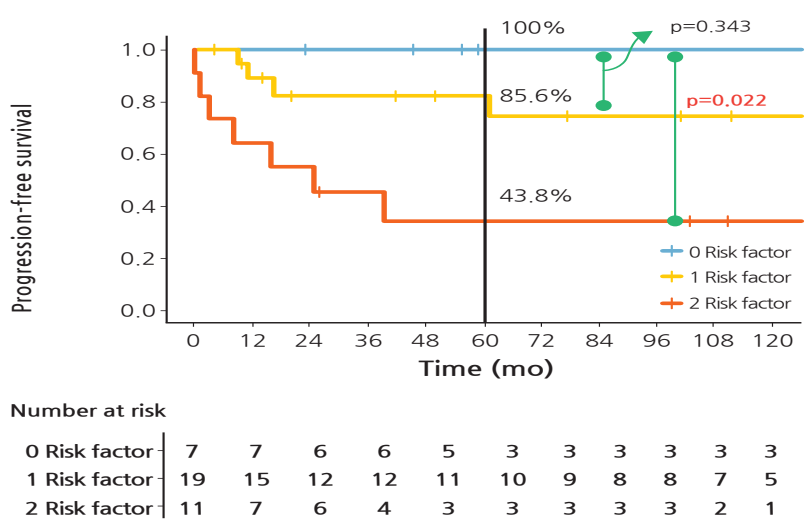

Fig. 2. Progression-free survival by the number of risk factors. p-values were estimated using log-rank test and compared with the number of risk factors $=0$.

\section{Survival outcomes and prognostic factors}

The median duration of follow-up was 105.9 months (range, 7.7 to 311.1 months). Five-year PFS rate was $70.9 \%$ (Fig. 1). Eight patients experienced in-field failure and 3 patients developed out-field failure. In univariate analysis (Table 2), administration of PORT at the initial diagnosis resulted in marginally improved PFS compared to deferring PORT after recurrence (hazard ratio $[\mathrm{HR}], 7.544 ;$ 95\% confidence interval [CI], 0.966-58.950; $\mathrm{p}=0.054)$. In addition, RT timing $>5.7$ weeks was also a marginally poor prognostic factor, with a $\mathrm{HR}$ of $3.373(95 \% \mathrm{Cl}$, 0.892-12.760; $p=0.073$ ). Tumor size, extent of surgery, margin status, or RT dose was not significant prognostic factor. On multivariate analysis (Table 2) involving administration of PORT at the initial diagnosis and RT timing, these two variables were all associated with PFS, with $\mathrm{HR}$ of $8.173(95 \% \mathrm{Cl}, 1.044$ 63.990; $p=0.045)$ and $3.698(95 \% \mathrm{Cl}, 0.975-14.020 ; p=0.054)$, 
Table 2. Univariate and multivariate analyses of progression-free survival using Cox proportional hazard model

\begin{tabular}{|c|c|c|c|c|c|}
\hline \multirow{2}{*}{ Variable } & \multirow{2}{*}{$\begin{array}{c}\text { No. of } \\
\text { patients }\end{array}$} & \multicolumn{2}{|c|}{ Univariate } & \multicolumn{2}{|c|}{ Multivariate } \\
\hline & & p-value & $\operatorname{HR}(95 \% \mathrm{Cl})$ & $p$-value & HR $(95 \% \mathrm{Cl})$ \\
\hline \multicolumn{6}{|l|}{ Sex } \\
\hline Male & 16 & 0.231 & Ref & & \\
\hline Female & 21 & & 2.255 (0.597-8.522) & & \\
\hline$A g e^{a)}$ & & 0.155 & $0.975(0.940-1.010)$ & & \\
\hline \multicolumn{6}{|l|}{ Location } \\
\hline Extra-abdomen & 29 & 0.998 & Ref & & \\
\hline Abdomen & 8 & & 3.318e-09 (0-infinity) & & \\
\hline Tumor size ${ }^{a)}$ & & 0.197 & 1.086 (0.958-1.231) & & \\
\hline \multicolumn{6}{|l|}{ Surgery } \\
\hline Wide excision & 15 & 0.446 & Ref & & \\
\hline Debulking & 22 & & $0.630(0.192-2.068)$ & & \\
\hline \multicolumn{6}{|l|}{ Margin } \\
\hline Negative & 8 & 0.716 & Ref & & \\
\hline Positive & 29 & & $1.330(0.287-6.166)$ & & \\
\hline \multicolumn{6}{|l|}{ PORT } \\
\hline At initial & 15 & 0.054 & Ref & 0.045 & Ref \\
\hline At recurrence & 22 & & $7.544(0.966-58.950)$ & & $8.173(1.044-63.990)$ \\
\hline RT dose $e^{a)}$ & & 0.152 & $0.999(0.998-1.000)$ & & \\
\hline \multicolumn{6}{|l|}{ RT timing ${ }^{b)}(w k)$} \\
\hline$\leq 5.7$ & 18 & 0.073 & Ref & 0.054 & Ref \\
\hline$>5.7$ & 19 & & $3.373(0.892-12.760)$ & & $3.698(0.975-14.020)$ \\
\hline RT duration $\left.{ }^{a, c}\right)$ & & 0.399 & $0.768(0.415-1.419)$ & & \\
\hline
\end{tabular}

$H R$, hazard ratio; $\mathrm{Cl}$, confidence interval; PORT, postoperative radiotherapy; $\mathrm{RT}$, radiotherapy.

${ }^{a}$ Incremental. ${ }^{b)}$ Interval from operation to radiotherapy. ${ }^{c)}$ Period between beginning and end of radiotherapy.

respectively.

We considered RT timing longer than 5.7 weeks and deferring PORT after recurrence as risk factors. PFS rates of 37 patients were then compared according to the number of risk factors (Fig. 2). Five-year PFS rates of patients with no risk factor and one risk factor were 100\% and $82.2 \%$, respectively, showing no significant difference between the two $(p=0.203)$. However, patients with 2 risk factors had significantly lower 5 -year PFS rate than patients with no risk factor (34.1\% vs. 100.0\%; $p=0.012$ ).

\section{Immunohistochemistry of $\beta$-catenin and SMA}

IHC staining intensities for $\beta$-catenin and SMA were available for 11 and 12 patients, respectively. Based on IHC intensity of nuclear $\beta$-catenin, 6 patients were included into the low intensity group while 5 were assigned into the high intensity group (Fig. 3A). The 5-year PFS rate was 100\% in the high intensity group and $62.5 \%$ in the low intensity group (Fig. 3B), showing no significant difference between the two $(p=0.260)$.
Seven patients had low staining intensity of SMA while five patients had high intensity. The 5-year PFS rate was 66.7\% regardless of its intensity $(p=0.700)$.

\section{Discussion and Conclusion}

In this study, local control rate for AF was comparable to that in other studies $[1,12-14]$. The longer the interval from surgery to PORT, the poorer the survival rate. Administration of PORT at initial diagnosis resulted in significantly improved PFS compared to deferring PORT after recurrence. Patients with higher nuclear $\beta$-catenin intensity tended to have better PFS.

In previous studies, the incidence of AF was so low that the treatment strategy was not unified among patients. Furthermore, some patients did not receive PORT while others received PORT [12]. With such differences, prognostic factors affecting survival may be different from study to study. Unlike other studies, we confined patients with AF to those who received $P O R T$. 
A

Low intensity

High intensity

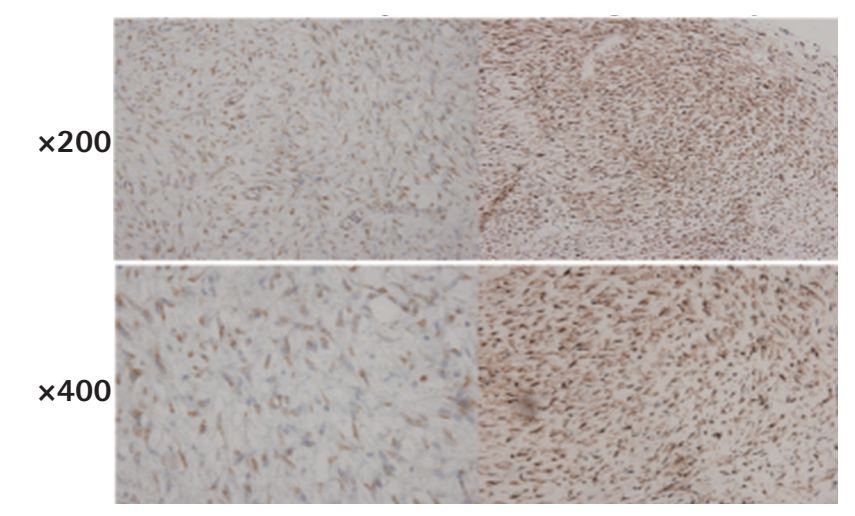

B

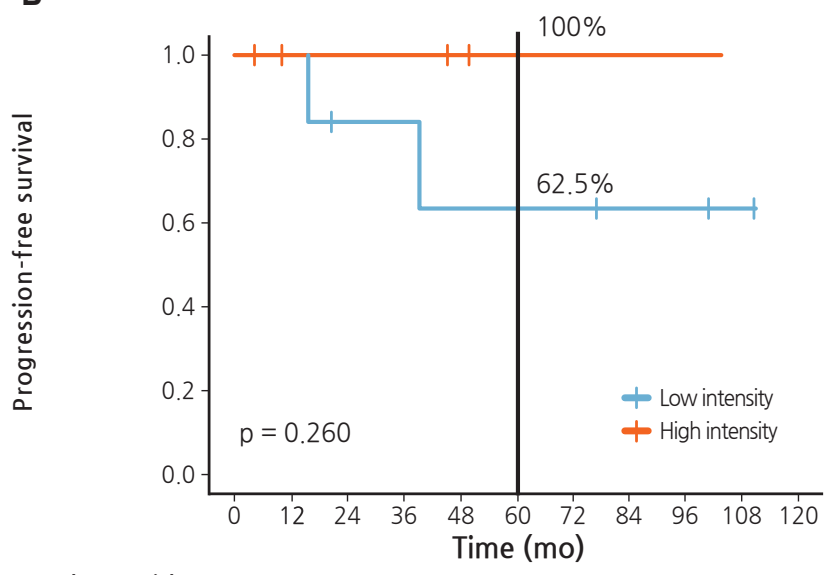

Number at risk

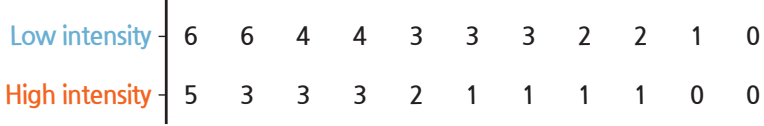

Fig. 3. Intensity classification of $\beta$-catenin immunohistochemistry (A) and comparison of progression-free survival according to $\beta$-catenin intensity using log-rank test (B). Low intensity group, negative or view nuclei at $\times 400$ to confirm nuclear accumulation $(n=6)$; high intensity group, view at $\times 200(n=5)$.

Almost $60 \%$ of patients underwent PORT after recurrence. The median number of relapses before receiving PORT was 2. At the time of initial diagnosis of AF, PORT was delivered to 15 patients due to positive resection margin $(n=13)$, close resection margin $(n=1)$, or large tumor size $(n=1 ; 13 \mathrm{~cm}$ in size). These represent the current clinical practice of deferring PORT unless there are risk factors for local recurrence.

To the best of our knowledge, this is the first study to examine the relationship between the onset of PORT and survival of patients with AF. Multivariate analysis showed that increased RT timing was associated with poorer PFS. This result might be explained by repopulation of microscopic residual disease after surgery. The reasons for delayed RT were wound problem in 3 patients and re-evaluation after surgery in 2 patients. One patient had delayed RT due to waiting for full engraftment. The other one was due to a change in the institution receiving RT. However, most of the patients who received RT after 5.7 weeks postoperatively started RT late for no apparent reason $(n=12 ; 63.2 \%)$. There should be a need to prepare for RT immediately after diagnosis of surgical specimens. Patients treated with PORT at initial diagnosis had better PFS than those with PORT after recurrence.

The resection margin status has been considered to be the most important prognostic factor for local control $[15,16]$. In particular, Ballo et al. [16] reported that because PORT for positive margin improved the prognosis, function-preserving surgery was appropriate. Contrary to expectations, our study showed no significant difference in PFS between negative and positive resection margins. Nine of 29 patients with positive resection margin developed disease progression or local recurrence. Among 8 patients with negative resection margin, 2 patients experienced local recurrence. Although the number of disease progression or recurrence in our study might be too small to obtain statistical power, Salas et al. [17] have also reported that resection margin does not consistently correlate with recurrence. They explained that AF was markedly infiltrative, making it difficult to assess resection margin [17].

Several studies have demonstrated that nuclear $\beta$-catenin expression evaluated by IHC is observed in more than $80 \%$ of patients with AF $[18,19]$. Similarly, nuclear $\beta$-catenin accumulation was recognized in $83 \%$ of available cases in our study. Gebert et al. [10] have proposed that nuclear $\beta$-catenin expression is associated with an increase in local recurrence. However, Lazar et al. [3] have reported that decreased rather than increased intensity of nuclear $\beta$-catenin is associated with more aggressive phenotype. Our results suggested a tendency of an inverse correlation between PFS and nuclear $\beta$-catenin expression as the result of Lazar et al. [3], although the correlation was not statistically significant in the present study. It was surprising that patients with high intensity of $\beta$-catenin did not experience any local failure. Possible explanation for this might be that nuclear translocation of $\beta$-catenin can promote cell proliferation and $\beta$-catenin protein levels are peak at G2/M phase of the cell cycle [20] which is known as the radiosensitive phase. Considering only 9 of 37 patients received PORT in the study of Gebert et al. [10], our results suggested that PORT might improve PFS in 
patients with AF expressing high nuclear $\beta$-catenin intensity. Further basic research is needed to evaluate the underlying mechanism.

Limitations of our study include its retrospective nature and a small number of patients. The long study inclusion period resulted in receiving PORT with heterogeneous techniques and doses. About half of patients underwent two-dimensional radiotherapy not used currently. Finally, external validation of grading system of $\mathrm{IHC}$ is required. Future prospective trials are needed. However, considering rare incidence of $A F$, such trials are difficult to be conducted.

In conclusion, PORT should be performed immediately after surgery irrespective of margin status or tumor size especially in recurrent case. Nuclear $\beta$-catenin staining intensity of IHC might be correlated with local recurrence. Further investigations are needed to validate its prognostic value.

\section{Conflict of Interest}

No potential conflict of interest relevant to this article was reported.

\section{References}

1. Harati $K_{1}$ Jaenisch $A$, Behr $B$, et al. Effect of surgical margins on prognosis in aggressive fibromatosis: A single-institutional analysis of 90 patients. Oncol Lett 2017;14:5129-34.

2. Kasper B, Baumgarten C, Garcia J, et al. An update on the management of sporadic desmoid-type fibromatosis: a European Consensus Initiative between Sarcoma PAtients EuroNet (SPAEN) and European Organization for Research and Treatment of Cancer (EORTC)/Soft Tissue and Bone Sarcoma Group (STBSG). Ann Oncol 2017;28:2399-408.

3. Lazar AJ, Tuvin D, Hajibashi $S$, et al. Specific mutations in the beta-catenin gene (CTNNB1) correlate with local recurrence in sporadic desmoid tumors. Am J Pathol 2008;173:1518-27.

4. Kamath SS, Parsons JT, Marcus RB, Zlotecki RA, Scarborough MT. Radiotherapy for local control of aggressive fibromatosis. Int J Radiat Oncol Biol Phys 1996;36:325-8.

5. Zlotecki RA, Scarborough MT, Morris CG, et al. External beam radiotherapy for primary and adjuvant management of aggressive fibromatosis. Int J Radiat Oncol Biol Phys 2002;54:177-81.

6. Willert $K_{1}$ Jones KA. Wnt signaling: is the party in the nucleus? Genes Dev 2006;20:1394-404.

7. Colombo $C$, Miceli $R$, Lazar AJ, et al. CTNNB1 $45 F$ mutation is a molecular prognosticator of increased postoperative primary desmoid tumor recurrence: an independent, multicenter validation study. Cancer 2013;119:3696-702.

8. Micke P, Ostman A. Exploring the tumour environment: cancer-associated fibroblasts as targets in cancer therapy. Expert Opin Ther Targets 2005;9:1217-33.

9. Olumi AF, Grossfeld GD, Hayward SW, Carroll PR, Tlsty TD, Cunha GR. Carcinoma-associated fibroblasts direct tumor progression of initiated human prostatic epithelium. Cancer Res 1999;59:5002-11.

10. Gebert C, Hardes J, Kersting C, et al. Expression of betacatenin and $p 53$ are prognostic factors in deep aggressive fibromatosis. Histopathology 2007;50:491-7.

11. Parikh JG, Kulkarni A, Johns C. -smooth muscle actin-positive fibroblasts correlate with poor survival in hepatocellular carcinoma. Oncol Lett 2014;7:573-5.

12. Mueller $C$, Croner $R$, Klein $P$, Grützmann $R$, Vassos N. Primary and recurrent sporadic desmoids: Prognostic factors influencing recurrence-free survival after complete gross resection. Int J Surg 2016;31:63-70.

13. Wirth $L$, Klein A, Baur-Melnyk $A$, et al. Desmoid tumours of the extremity and trunk: a retrospective study of 44 patients. BMC Musculoskelet Disord 2018;19:2.

14. Santti K, Beule A, Tuomikoski L, et al. Radiotherapy in desmoid tumors: treatment response, local control, and analysis of local failures. Strahlenther Onkol 2017;193:269-75.

15. Goy BW, Lee SP, Eilber $F$, et al. The role of adjuvant radiotherapy in the treatment of resectable desmoid tumors. Int J Radiat Oncol Biol Phys 1997;39:659-65.

16. Ballo MT, Zagars GK, Pollack A, Pisters PW, Pollack RA. Desmoid tumor: prognostic factors and outcome after surgery, radiation therapy, or combined surgery and radiation therapy. J Clin Oncol 1999;17:158-67.

17. Salas S, Dufresne A, Bui B, et al. Prognostic factors influencing progression-free survival determined from a series of sporadic desmoid tumors: a wait-and-see policy according to tumor presentation. J Clin Oncol 2011;29:3553-8.

18. Carlson JW, Fletcher CD. Immunohistochemistry for beta-catenin in the differential diagnosis of spindle cell lesions: analysis of a series and review of the literature. Histopathology 2007;51:509-14.

19. Ng TL, Gown AM, Barry TS, et al. Nuclear beta-catenin in mesenchymal tumors. Mod Pathol 2005;18:68-74.

20. Orford K, Orford CC, Byers SW. Exogenous expression of betacatenin regulates contact inhibition, anchorage-independent growth, anoikis, and radiation-induced cell cycle arrest. J Cell Biol 1999;146:855-68. 